\title{
Classification of Quaternary Hermitian Self-Dual Codes of Length 20
}

\author{
Masaaki Harada*† and Akihiro Munemasa ${ }^{\ddagger}$
}

June 5, 2018

\begin{abstract}
A classification of quaternary Hermitian self-dual codes of length 20 is given. Using this classification, a classification of extremal quaternary Hermitian selfdual codes of length 22 is also given.
\end{abstract}

\section{Introduction}

Let $\mathbb{F}_{4}=\{0,1, \omega, \bar{\omega}\}$ be the finite field of order four, where $\bar{\omega}=\omega^{2}=\omega+1$. Codes over $\mathbb{F}_{4}$ are often called quaternary. All codes in this note are quaternary. The Hermitian dual code $C^{\perp}$ of a code $C$ of length $n$ is defined as $C^{\perp}=\left\{x \in \mathbb{F}_{4}^{n} \mid x \cdot c=0\right.$ for all $c \in$ $C\}$, where $x \cdot y=\sum_{i=1}^{n} x_{i} y_{i}{ }^{2}$ for $x=\left(x_{1}, \ldots, x_{n}\right), y=\left(y_{1}, \ldots, y_{n}\right) \in \mathbb{F}_{4}^{n}$, which is known as the Hermitian inner product. A code $C$ is called Hermitian self-orthogonal if $C \subset C^{\perp}$, and $C$ is called Hermitian self-dual if $C=C^{\perp}$. It is known that an $[n, k]$ code $C$ is Hermitian self-dual if and only if the weights of all codewords of $C$ are even, that is, $C$ is even, and $n=2 k$ [1]. It was shown in [1] that the minimum weight $d$ of a Hermitian self-dual code of length $n$ is bounded by $d \leq 2\lfloor n / 6\rfloor+2$. A Hermitian self-dual code of length $n$ and minimum weight $d=2\lfloor n / 6\rfloor+2$ is called extremal.

Two codes $C$ and $C^{\prime}$ are equivalent if there is some monomial matrix $M$ over $\mathbb{F}_{4}$ such that $C^{\prime}=C M=\{c M \mid c \in C\}$ [1]. A monomial matrix which maps $C$ to itself is called an automorphism of $C$, and the set of all automorphisms of $C$ forms the automorphism group $\operatorname{Aut}(C)$ of $C$. Clearly, $\omega I$ and $\bar{\omega} I$ are elements of $\operatorname{Aut}(C)$, where $I$ denotes the identity matrix, so $\{I, \omega I, \bar{\omega} I\}$ is the smallest possible automorphism group. Such an automorphism group is called trivial.

The classification of Hermitian self-dual codes was begun by [1] and the classification for lengths up to 14 was done in [1]. The classification is extended to length

*This work of the first author was supported by JST PRESTO program.

${ }^{\dagger}$ M. Harada is with the Department of Mathematical Sciences, Yamagata University, Yamagata 990-8560, Japan, and PRESTO, Japan Science and Technology Agency (JST), Kawaguchi, Saitama 332-0012, Japan email: mharada@sci.kj.yamagata-u.ac.jp.

${ }^{\ddagger}$ A. Munemasa is with the Graduate School of Information Sciences, Tohoku University, Sendai 980-8579, Japan email: munemasa@math.is.tohoku.ac.jp 
18 [2, 3]. At length 20, a classification of extremal Hermitian self-dual codes was completed [4] under a weaker equivalence (see Subsection 3.3 for the definition). At length 22, at least 46 inequivalent extremal self-dual codes are known [5]. In Table 1, the numbers $\#_{d}$ of inequivalent Hermitian self-dual codes with minimum weight $d$ are given along with references.

Table 1: Hermitian self-dual codes

\begin{tabular}{|c|cccc|cc|c|c|}
\hline & \multicolumn{3}{|c|}{ Indecomposable } & \multicolumn{2}{c|}{ Decomposable } & & \\
$n$ & $\#_{2}$ & $\#_{4}$ & $\#_{6}$ & $\#_{8}$ & $\#_{2}$ & $\#_{4}$ & Total & References \\
\hline 2 & 1 & & & & & & 1 & {$[1]$} \\
4 & 0 & & & & 1 & & 1 & {$[1]$} \\
6 & 0 & 1 & & & 1 & & 2 & {$[1]$} \\
8 & 0 & 1 & & & 2 & & 3 & {$[1]$} \\
10 & 0 & 2 & & & 3 & & 5 & {$[1]$} \\
12 & 0 & 4 & & & 5 & 1 & 10 & {$[1]$} \\
14 & 0 & 9 & 1 & & 10 & 1 & 21 & {$[1]$} \\
16 & 0 & 27 & 4 & & 21 & 3 & 55 & {$[2]$} \\
18 & 0 & 152 & 30 & 1 & 55 & 7 & 245 & {$[3,[4]$} \\
\hline 20 & 0 & 2163 & 999 & 2 & 245 & 18 & 3427 & {$[4]$, Section $[3]$} \\
22 & 0 & $?$ & $?$ & 723 & 3427 & 52 & $?$ & Section $[4$ \\
\hline
\end{tabular}

The main aim of this note is to give a complete classification of Hermitian self-dual codes of length 20 .

Theorem 1. There are 3427 inequivalent Hermitian self-dual codes of length 20. Of these two are extremal, 999 have minimum weight 6, 2181 have minimum weight 4, and 245 have minimum weight 2.

The method used, which is similar to that given in [4, is described in Subsection 2.1.

From the classification of Hermitian self-dual $[20,10,6]$ codes, we also classify extremal Hermitian self-dual codes of length 22 .

It has been a question to determine if there is a Hermitian self-dual code with a trivial automorphism group (see [1, Open Problem (4)]). There is no Hermitian self-dual code with a trivial automorphism group for lengths up to 18 (see [1, 2, 3]). From our classification given in Section 3, we see that such a code exists at length 20.

Generator matrices of all Hermitian self-dual codes of length 20 and all extremal Hermitian self-dual codes of length 22 can be obtained electronically from [6]. All computer calculations in this note were done by Magma [7]. 


\section{Preliminaries}

\subsection{Classification method}

Here we describe a method for classifying Hermitian self-dual codes. This method is similar to that given in [4].

Suppose that $C$ is a Hermitian self-dual $[n, n / 2, d]$ code with $d \geq 4$. Define a subcode of $C$ as follows

$$
C_{0}=\left\{\left(x_{1}, x_{2}, \ldots, x_{n}\right) \in C \mid x_{n-1}=x_{n}\right\} .
$$

Since $C^{\perp}$ has no codeword of weight $2, C_{0}$ has dimension $n / 2-1$. We may assume that there is a codeword $x=\left(x_{1}, \ldots, x_{n}\right)$ of weight $d$ in $C$ with $x_{n-1}=x_{n} \neq 0$. Then, the following code

$$
C_{1}=\left\{\left(x_{1}, x_{2}, \ldots, x_{n-2}\right) \mid\left(x_{1}, x_{2}, \ldots, x_{n}\right) \in C_{0}\right\}
$$

is a self-dual $[n-2, n / 2-1, d-2]$ code. Thus, the subcode $C_{0}$ has generator matrix of the form

$$
G_{0}=\left(\begin{array}{l|cc} 
& a_{1} & a_{1} \\
G_{1} & \vdots & \vdots \\
& a_{n / 2-1} & a_{n / 2-1}
\end{array}\right),
$$

where $G_{1}$ is a generator matrix of $C_{1}$ and $a_{i} \in \mathbb{F}_{4}(i=1, \ldots, n / 2-1)$. It follows that any Hermitian self-dual $[n, n / 2, d]$ code is constructed as the code $\left\langle C_{0}, x\right\rangle$ for some code $C_{0}$ with generator matrix of the form (11) and some vector $x \in C_{0}^{\perp} \backslash C_{0}$, where $\left\langle C_{0}, x\right\rangle$ denotes the code generated by the codewords of $C_{0}$ and $x$.

In this way, all Hermitian self-dual $[n, n / 2, d]$ codes, which must be checked further for equivalence, are constructed, by taking generator matrices of all inequivalent selfdual $[n-2, n / 2-1, d-2]$ codes as matrices $G_{1}$, and by considering $a_{1} \in\{0,1\}$ and $a_{i} \in \mathbb{F}_{4}(i=2, \ldots, n / 2-1)$ in (11). As described in [4], the number of possibilities for $a_{i}(i=2, \ldots, n / 2-1)$ is decreased by applying elements of $\operatorname{Aut}\left(C_{1}\right)$ to the first $n-2$ coordinates of (11).

\subsection{Mass formula for weight enumerators}

Now we give a mass formula for weight enumerators of Hermitian self-dual codes.

Lemma 2. Let $n$ be an even positive integer. Let $W_{C}(y)$ denote the weight enumerator of a code $C$. Then

$$
\begin{aligned}
\sum_{C} W_{C}(y)= & \\
& \prod_{i=0}^{n / 2-1}\left(2^{2 i+1}+1\right)+\sum_{j=1}^{n / 2}\left(\begin{array}{c}
n \\
2 j
\end{array}\right) 3^{2 j} \prod_{i=0}^{n / 2-2}\left(2^{2 i+1}+1\right) y^{2 j},
\end{aligned}
$$

where $C$ runs through the set of all Hermitian self-dual codes of length $n$. 
Proof. Let $\operatorname{wt}(x)$ denote the weight of a vector $x \in \mathbb{F}_{4}^{n}$.

$$
\begin{aligned}
& \sum_{C} W_{C}(y) \\
& =\sum_{j=0}^{n / 2} \sum_{C} \#\{x \in C \mid \operatorname{wt}(x)=2 j\} y^{2 j} \\
& =\sum_{j=0}^{n / 2} \sum_{\substack{x \in \mathbb{F}_{4}^{n} \\
\mathrm{wt}(x)=2 j}} \#\{C \mid x \in C\} y^{2 j} \\
& =\prod_{i=0}^{n / 2-1}\left(2^{2 i+1}+1\right)+\sum_{j=1}^{n / 2} \sum_{\mathrm{wt}(x)=2 j} \#\{C \mid x \in C\} y^{2 j}
\end{aligned}
$$

(by [1, Theorem 19])

$$
=\prod_{i=0}^{n / 2-1}\left(2^{2 i+1}+1\right)+\sum_{j=1}^{n / 2} \sum_{\mathrm{wt}(x)=2 j} \prod_{i=0}^{n / 2-2}\left(2^{2 i+1}+1\right) y^{2 j}
$$

(by [1, Theorem 23])

$$
=\prod_{i=0}^{n / 2-1}\left(2^{2 i+1}+1\right)+\sum_{j=1}^{n / 2}\left(\begin{array}{c}
n \\
2 j
\end{array}\right) 3^{2 j} \prod_{i=0}^{n / 2-2}\left(2^{2 i+1}+1\right) y^{2 j} \text {. }
$$

Lemma 3. Let $n$ and $d$ be even positive integers. Let $\mathcal{C}$ be a family of pairwise inequivalent Hermitian self-dual codes of length $n$ with minimum weight at most $d$. Then $\mathcal{C}$ is a complete set of representatives for equivalence classes of Hermitian selfdual codes of length $n$ with minimum weight at most $d$, if and only if

$$
\begin{aligned}
\sum_{C \in \mathcal{C}} \frac{3^{n} n !}{\# \operatorname{Aut}(C)} \#\{x \in C \mid \operatorname{wt}(x)=d\} & \\
& =\left(\begin{array}{l}
n \\
d
\end{array}\right) 3^{d} \prod_{i=0}^{n / 2-2}\left(2^{2 i+1}+1\right) .
\end{aligned}
$$

Proof. Consider the coefficient of $y^{d}$ in the formula (2) in Lemma 2 .

\section{Classification of self-dual codes of length 20}

In this section, we give a classification of Hermitian self-dual codes of length 20 .

\subsection{Decomposable codes}

We first consider decomposable Hermitian self-dual codes. By Theorem 28 in [1], any Hermitian self-dual code with minimum weight 2 is decomposable as $C_{2} \oplus C_{18}$, where 
$C_{2}$ is the unique Hermitian self-dual code of length 2 and $C_{18}$ is some Hermitian selfdual code of length 18. Hence, from Table 1, there are 245 inequivalent Hermitian self-dual codes with minimum weight 2. Every decomposable Hermitian self-dual code with minimum weight 4 is a direct sum of indecomposable codes of length at least 6 , since there are no Hermitian self-dual code with minimum weight at least 4 for lengths less than 6. From Table 1, the numbers of indecomposable Hermitian self-dual codes of lengths $6,8,10,12,14$ are $1,1,2,4,10$, respectively. It follows that the numbers of Hermitian self-dual codes of the forms $C_{6} \oplus C_{6} \oplus C_{8}, C_{6} \oplus C_{14}, C_{8} \oplus C_{12}$ or $C_{10} \oplus C_{10}$, where $C_{n}$ denotes an indecomposable code of length $n$, are $1,10,4,3$, respectively. Therefore, there are 18 inequivalent decomposable Hermitian self-dual codes with minimum weight 4 . There is no decomposable Hermitian self-dual code with minimum weight $d \geq 6$.

\subsection{Indecomposable codes}

From the set of inequivalent Hermitian self-dual $[18,9,2]$ codes given in [3], the method given in Subsection 2.1 allows to enumerate the set $\mathcal{C}_{20,4}$ of the 2181 inequivalent Hermitian self-dual $[20,10,4]$ codes. Let $\mathcal{C}_{20,2}$ denote the set of the 245 inequivalent Hermitian self-dual codes of length 20 and minimum weight 2. Setting $\mathcal{C}=\mathcal{C}_{20,2} \cup \mathcal{C}_{20,4}$ in Lemma 3, one can verify that there is no other Hermitian self-dual $[20,10,4]$ code, by calculating the summand in the right-hand side of (3) for all codes of $\mathcal{C}$. Similarly, we found the set of 999 (resp. 2) inequivalent Hermitian self-dual codes with minimum weight 6 (resp. 8). In this way, we found the set $\mathcal{C}_{20}$ of 3182 inequivalent Hermitian self-dual codes with minimum weight $d \geq 4$ satisfying

$$
\sum_{C \in \mathcal{C}_{20} \cup \mathcal{C}_{20,2}} \frac{3^{20} \cdot 20 !}{\# \operatorname{Aut}(C)}=2229034892015508532492061011707,
$$

which is the constant term of (2). This constant term $\prod_{i=0}^{9}\left(2^{2 i+1}+1\right)$ gives the number of the distinct self-dual codes of length 20 . The mass formula shows that there is no other Hermitian self-dual code of length 20 and the classification is complete. Therefore, we have Theorem 1 .

This computation was performed in MAgma [7. In principle, such a computation can be done by classifying Hermitian self-dual codes by the Magma function IsIsomorphic, then their automorphism groups can be calculated by AutomorphismGroup. The orders of the automorphism groups of the 3427 codes are listed in Table 2, where (\# Aut, $N$ (\# Aut)) lists the number $N$ (\# Aut) of the codes with an automorphism group of order \# Aut.

Alternatively, the set of 3182 inequivalent Hermitian self-dual codes of length 20 and minimum weight $d \geq 4$ can be found by a method similar to the one given in [3] as follows. Recall that two self-dual codes $C$ and $C^{\prime}$ of length $n$ are said to be neighbors if the dimension of $C \cap C^{\prime}$ is $n / 2-1$. Let $D_{20}$ be the extremal Hermitian self-dual code of length 20 generated by the second generator matrix in [4, Fig. 4]. Let $N_{0}=\left\{D_{20}\right\}$ and $N_{i+1}=\bigcup_{C \in N_{i}} \operatorname{Nei}(C)(i=0,1,2,3)$, where Nei $(C)$ denotes the set of inequivalent Hermitian self-dual neighbors with minimum weight $d \geq 4$ of $C$. 
Table 2: Orders of the automorphism groups for length 20

\begin{tabular}{|c|c|}
\hline$d$ & (\# Aut, $N(\#$ Aut $))$ \\
\hline 8 & $(4320,1),(5760,1)$ \\
\hline 6 & $\begin{array}{l}(3,419),(6,328),(9,13),(12,103),(15,1),(18,26),(24,15),(27,1),(30,5),(36,33),(48,9),(54,6),(60,3),(72,11),(96,2), \\
(108,4),(144,3),(192,3),(216,2),(288,4),(360,2),(384,1),(576,1),(1440,1),(2160,1),(3456,1),(5760,1)\end{array}$ \\
\hline 4 & $\begin{array}{l}(12,377),(24,258),(36,22),(48,363),(72,38),(96,134),(144,41),(192,176),(216,4),(240,3),(288,60),(384,82),(432,13), \\
(576,31),(720,1),(768,44),(864,9),(1008,1),(1080,2),(1152,92),(1296,3),(1440,3),(1536,30),(1728,9),(2160,3), \\
(2304,42),(2592,1),(2880,3),(3072,6),(3456,9),(3600,1),(4320,2),(4608,31),(5184,3),(5760,4),(6144,1),(6480,1), \\
(6912,42),(7560,2),(8640,1),(9216,22),(10368,3),(10800,1),(11520,3),(12288,1),(13824,18),(15360,1),(16128,1), \\
(17280,9),(18432,3),(20736,1),(23040,4),(24576,1),(25920,1),(27648,9),(34560,1),(36288,1),(36864,2),(39312,1), \\
(41472,8),(48384,2),(51840,1),(55296,21),(69120,8),(82944,7),(92160,1),(96768,1),(103680,3),(110592,3),(124416,1), \\
(138240,3),(145152,1),(165888,4),(184320,1),(193536,1),(207360,4),(221184,4),(248832,2),(290304,1),(331776,3), \\
(345600,1),(368640,2),(387072,1),(414720,2),(518400,1),(552960,3),(580608,2),(622080,2),(663552,5),(725760,1), \\
(777600,1),(829440,5),(884736,2),(1105920,2),(1161216,1),(1244160,1),(1382400,1),(1658880,1),(1866240,1), \\
(2073600,2),(2488320,1),(3110400,1),(3538080,1),(3732480,1),(4064256,1),(4147200,2),(4354560,1),(5806080,1), \\
(6635520,1),(7464960,1),(10886400,1),(11612160,1),(13271040,1),(13934592,1),(14929920,1),(15552000,1), \\
(22118400,1),(23224320,1),(37324800,1),(41472000,1),(41803776,1),(58060800,1),(66355200,1),(121927680,1), \\
(124416000,1),(182891520,1),(278691840,1),(279936000,1),(933120000,1),(1045094400,1),(5573836800,1), \\
(9405849600,1)\end{array}$ \\
\hline 2 & $\begin{array}{l}(36,1),(72,5),(108,1),(144,9),(162,1),(216,6),(288,4),(324,2),(432,7),(576,8),(864,5),(1080,2),(1152,9),(1296,2), \\
(1728,10),(2304,6),(2592,1),(3024,1),(3456,3),(3888,1),(4608,2),(5184,2),(6480,1),(6912,13),(7776,1),(9216,1), \\
(10368,3),(13824,6),(17496,1),(18432,1),(20736,3),(25920,1),(27648,4),(31104,1),(34560,1),(36864,1),(41472,7), \\
(55296,1),(62208,2),(72576,1),(77760,1),(82944,8),(84240,1),(103680,2),(110592,3),(124416,2),(139968,1), \\
(146880,1),(165888,4),(207360,2),(248832,1),(290304,1),(311040,1),(331776,4),(373248,1),(414720,1),(497664,3), \\
(622080,1),(746496,1),(829440,2),(870912,1),(995328,3),(1244160,3),(1327104,3),(1492992,4),(2239488,1), \\
(2488320,1),(3110400,1),(3317760,1),(3732480,1),(3981312,1),(4245696,1),(4354560,1),(4478976,1),(5806080,1), \\
(6635520,1),(6967296,1),(8957952,1),(9953280,2),(11943936,1),(12192768,1),(15925248,1),(17915904,1),(18662400,1), \\
(22394880,1),(24883200,3),(26127360,1),(37324800,1),(44789760,1),(67184640,1),(69984000,1),(107495424,1), \\
(139345920,1),(195955200,1),(219469824,1),(322486272,1),(335923200,1),(447897600,2),(522547200,1),(940584960,1), \\
(1114767360,1),(1254113280,1),(1672151040,1),(1679616000,1),(2149908480,1),(2341011456,1),(5374771200,1), \\
(5643509760,1),(20155392000,1),(45349632000,1),(72559411200,1),(135444234240,1),(1523747635200,1), \\
(219419659468800,1)\end{array}$ \\
\hline
\end{tabular}

Then the set

$$
N_{0} \cup N_{1} \cup N_{2} \cup N_{3} \cup N_{4},
$$

contains 3182 inequivalent Hermitian self-dual codes of length 20 and minimum weight $d \geq 4$.

\subsection{A weaker equivalence}

In the above classification, we employ monomial matrices over $\mathbb{F}_{4}$ in the definition for equivalence of codes. To define a weaker equivalence, one could consider a conjugation $\gamma$ of $\mathbb{F}_{4}$ sending each element to its square, in the definition of equivalence, that is, two codes $C$ and $C^{\prime}$ are weakly equivalent if there is some monomial matrix $M$ over $\mathbb{F}_{4}$ such that $C^{\prime}=C M$ or $C^{\prime}=C M \gamma$ (see [4]). In fact, the classification of extremal self-dual codes of length 20 in [4] was done under the weaker equivalence. Our classification shows that the equivalence classes of such codes are the same under both definitions.

We have verified that there are 15, 636 and 323 pairs of self-dual codes with minimum weights 2,4 and 6, respectively, under the weaker equivalence. Hence, there are $3427-(15+636+323)=2453$ self-dual codes under the weaker equivalence for length 20 .

\section{Classification of extremal self-dual codes of length 22}

From the set of inequivalent Hermitian self-dual $[20,10,6]$ codes classified in the 
Table 3: Orders of the automorphism groups for length 22

\begin{tabular}{|l|}
\hline \multicolumn{1}{|c|}{ (\# Aut, $N$ (\# Aut)) } \\
\hline$(3,308),(6,229),(9,8),(12,73),(18,12),(24,39),(30,2),(36,19),(48,1)$, \\
$(60,1),(66,2),(72,5),(108,1),(120,1),(180,3),(192,1),(240,1),(288,4)$, \\
$(324,1),(360,1),(384,2),(504,2),(864,2),(1728,2),(17280,2),(1330560,1)$ \\
\hline
\end{tabular}

previous section, the method given in Subsection 2.1 allows to enumerate extremal Hermitian self-dual codes of length 22.

For every self-dual $[20,10,6]$ code, we have verified that the subcode generated by codewords of weight 6 has dimension at least 4 . Thus, we may assume that the first four rows of a generator matrix $G_{1}$ in (1) have weight 6 . This yields that $a_{1}=1$ and $a_{i} \in\{1, \omega, \bar{\omega}\}(i=2,3,4)$. From the 999 self-dual $[20,10,6]$ codes, 723 inequivalent extremal self-dual codes of length 22 are obtained. Therefore, we have the following:

Proposition 4. There are 723 inequivalent extremal Hermitian self-dual codes of length 22.

The orders of the automorphism groups of the 723 codes are listed in Table 3 , where (\# Aut, $N$ (\# Aut)) lists the number $N$ (\# Aut) of the codes with an automorphism group of order \# Aut. The code with an automorphism group of order 1330560 is equivalent to the code $C_{22, P 1}$ in [8, Table 2], which is one of the three inequivalent pure double circulant extremal self-dual codes of length 22 . We have verified that the automorphism group is isomorphic to the direct product of the Mathieu group $M_{22}$ of degree 22 and the cyclic group of order 3.

We have verified that there are 301 pairs of extremal self-dual codes of length 22, under the weaker equivalence given in Subsection 3.3. Hence, there are $723-301=$ 422 extremal self-dual codes of length 22 , under the weaker equivalence. We have also verified that the 422 extremal self-dual codes have different numbers $\left(B_{0}, B_{1}, \ldots, B_{22}\right)$, where $B_{j}$ denotes the number of distinct cosets of weight $j$. This shows that the 422 extremal self-dual codes are certainly inequivalent.

The smallest possible automorphism group is of order 3. From the constant term of (2), the number of inequivalent self-dual codes of length 22 is at least

$$
\frac{\prod_{i=0}^{10}\left(2^{2 i+1}+1\right)}{22 ! \cdot 3^{21}}>397588 .
$$

\section{Trivial automorphism groups}

It has been a question to determine if there is a Hermitian self-dual code with a trivial automorphism group (see [1, Open Problem (4)]). There is no Hermitian self-dual code with a trivial automorphism group for lengths up to 18 (see [1, 2, 3]). From our classification given in Section 3, we see that such a code exists at length 20 (see Table 21). Hence, we have an answer to the above problem. 


$$
M=\left(\begin{array}{ccccccccccc}
1 & 0 & 1 & 0 & 1 & 0 & \omega & \bar{\omega} & \omega & \bar{\omega} & 0 \\
\omega & \omega & 0 & \omega & 1 & 0 & \omega & \omega & \bar{\omega} & \omega & 1 \\
\bar{\omega} & \omega & \bar{\omega} & 0 & 0 & \bar{\omega} & \omega & \bar{\omega} & \bar{\omega} & 1 & \omega \\
\bar{\omega} & 0 & 1 & 1 & 0 & \omega & \bar{\omega} & 1 & \omega & \omega & \omega \\
\omega & \omega & 0 & \omega & \bar{\omega} & 1 & \bar{\omega} & \bar{\omega} & \bar{\omega} & 0 & \bar{\omega} \\
1 & \bar{\omega} & 1 & 0 & 0 & \omega & 0 & \bar{\omega} & 0 & \bar{\omega} & 1 \\
0 & 1 & \bar{\omega} & 0 & 0 & \omega & 1 & 0 & 1 & \omega & \bar{\omega} \\
\omega & 0 & \bar{\omega} & \bar{\omega} & \bar{\omega} & 1 & \bar{\omega} & 0 & \omega & \bar{\omega} & \bar{\omega} \\
1 & \omega & \omega & \omega & 1 & 1 & 0 & \bar{\omega} & 1 & 0 & \omega \\
1 & \omega & \bar{\omega} & \omega & \bar{\omega} & 1 & 1 & \bar{\omega} & 0 & \omega & 0 \\
0 & 0 & \omega & \bar{\omega} & \bar{\omega} & \omega & 1 & 1 & 1 & \omega & \omega
\end{array}\right)
$$

Figure 1: An extremal self-dual $[22,11,8]$ code with a trivial automorphism group

Corollary 5. The smallest length for which there is a quaternary Hermitian self-dual code with a trivial automorphism group is 20 .

We describe here an example of a code satisfying the conditions of Corollary 5 . Let $B_{20}$ be the pure double circulant code with generator matrix $(I, R)$, where $R$ is the circulant matrix with first row $(\bar{\omega}, 0, \omega, 0, \omega, 0, \bar{\omega}, 1, \omega, 1)$. The following code

$$
C_{20}=\left\langle B_{20} \cap\langle v\rangle^{\perp}, v\right\rangle
$$

where $v=(\omega, 1,1,1,1,1,1,1,1,1,0, \bar{\omega}, 0,0,1,0, \omega, \bar{\omega}, 1, \bar{\omega})$, is a Hermitian self-dual code of length 20 with a trivial automorphism group.

In addition, there is no extremal Hermitian self-dual code with a trivial automorphism group for length 20, but there are 308 extremal Hermitian self-dual codes with trivial automorphism groups for length 22 (see Tables 2 and 3). Hence, the smallest length for which there is a quaternary extremal Hermitian self-dual code with a trivial automorphism group is 22. For example, the code with generator matrix $(I, M)$ is an extremal self-dual $[22,11,8]$ code with a trivial automorphism group, where $M$ is given in Figure 1.

\section{References}

[1] F. J. MacWilliams, A. M. Odlyzko, N. J. A. Sloane, and H. N. Ward, "Self-dual codes over GF(4)," Journal of Combinatorial Theory Series A, vol. 25, no. 3, pp. 288-318, Nov 1978. http://linkinghub.elsevier.com/retrieve/pii/0097316578900213

[2] J. H. Conway, V. Pless, and N. J. A. Sloane, "Self-dual codes over $\mathrm{GF}(3)$ and $\mathrm{GF}(4)$ of length not exceeding 16," IEEE Transactions on Information Theory, vol. 25, no. 3, pp. 312-322, May 1979. http://ieeexplore.ieee.org/lpdocs/epic03/wrapper.htm?arnumber $=1056047$ 
[3] M. Harada, C. Lam, A. Munemasa, and V. D. Tonchev, "Classification of generalized Hadamard matrices $H(6,3)$ and quaternary hermitian self-dual codes of length 18," submitted. http://arxiv.org/abs/1007.2555v1

[4] W. C. Huffman, "Characterization of quaternary extremal codes of lengths 18 and 20," IEEE Transactions on Information Theory, vol. 43, no. 5, pp. 1613-1616, 1997. http://ieeexplore.ieee.org/lpdocs/epic03/wrapper.htm?arnumber $=623160$

[5] J.-L. Kim, "New self-dual codes over GF(4) with the highest known minimum weights," IEEE Transactions on Information Theory, vol. 47, no. 4, pp. 1575-1580, May 2001. http://ieeexplore.ieee.org/lpdocs/epic03/wrapper.htm?arnumber=923739

[6] M. Harada and A. Munemasa, "Database of self-dual codes." http://www.math.is.tohoku.ac.jp/ \{\}munemasa/selfdualcodes.htm

[7] W. Bosma and J. Cannon, "Handbook of magma functions." http://magma.maths.usyd.edu.au/magma/

[8] H. Miyabayashi, "A classification of double circulant hermitian self-dual codes over $\mathbb{F}_{4}$ of lengths up to 26," Bull. Yamagata Univ. Natural Sci., vol. 16, pp. 81-91, 2007. 